\title{
Safety and outcomes of endoscopic ultrasound-guided drainage for malignant biliary obstruction using cautery-enabled lumen- apposing metal stent
}

다(1)우우

\author{
Authors \\ Jerry Yung-Lun Chin, Samir Seleq, Frank Weilert \\ Institution \\ Department of Gastroenterology and Hepatology, Waikato \\ Hospital, Hamilton, New Zealand
}

submitted 9.5.2020

accepted after revision $\quad 15.7 .2020$

Bibliography

Endoscopy International Open 2020; 08: E1633-E1638

DOI 10.1055/a-1236-3217

ISSN 2364-3722

(c) 2020. The Author(s).

This is an open access article published by Thieme under the terms of the Creative Commons Attribution-NonDerivative-NonCommercial License, permitting copying and reproduction so long as the original work is given appropriate credit. Contents may not be used for commecial purposes, or adapted, remixed, transformed or built upon. (https://creativecommons.org/licenses/by-nc-nd/4.0/)

\section{Corresponding author}

Frank Weilert BSc, MBBCh, FRACP and FASGE, Department of Gastroenterology and Hepatology, Waikato Hospital, Pembroke Street, Hamilton, 3240, New Zealand

Fax: +64 78398899

Frank.Weilert@waikatodhb.health.nz

\section{ABSTRACT}

Background and study aims There is increasing evidence to suggest that EUS-guided biliary drainage (EUS-BD) is a safe and effective treatment alternative for patients with malignant biliary obstructions (MBOs) after failed endoscopic retrograde cholangiopancreatography.

Patients and methods We performed a retrospective analysis of data prospectively collected from patients with MBO who underwent choledochoduodenostomy (CDS) or gallbladder drainage (GBD) between August 2016 and June 2020 using the electrocautery-enabled lumen-apposing metal stents (ECE-LAMS). The primary endpoint was technical and clinical success. Secondary endpoints were adverse events (AEs) and reinterventions.

Results A total of 60 patients were included in the study, with 56 CDS and 4 GBD. Median age was 76 years with $57 \%$ male (34/60). The most common indication for EUS-BD was pancreatic cancer (78\%). Technical success was achieved in $100 \%$ of cases, with a clinical success rate of $91.7 \%$. Mean total bilirubin pre-procedure was $202 \mathrm{umol} / \mathrm{L}$ (normal $<20 \mathrm{umol} / \mathrm{L}$ ) and $63.8 \mathrm{umol} / \mathrm{L}$ post procedure $(P<.001)$. Twenty-one patients had bilirubin recorded at 2 weeks post EUS-BD with 20 of 21 patients demonstrating $>50 \%$ reduction in bilirubin (mean bilirubin reduction $75 \%$ ). AEs occurred in 12 of 60 patients (20\%), all of which were mild. The reintervention rate was $11.7 \%(7 / 60)$. Stent occlusion occurred in 10 of 60 patients $(16.7 \%)$ with a mean time to stent occlusion of 46.2 days (3-133). Stent patency of $83.3 \%$ was observed with a mean follow up of 7.9 months.

Conclusion EUS-CDS and GBD using ECE-LAMS are effective EUS-based techniques for managing patients with MBO. AEs are usually mild and resolved by reintervention.

\section{Introduction}

Malignant biliary obstruction (MBO) is conventionally treated with endoscopic retrograde cholangiopancreatography (ERCP) and placement of either plastic or metal stents. ERCP remains the gold standard in treatment of biliary obstruction, however, failed biliary cannulation is observed in up to $10 \%$ of cases, especially in patients with low performance status [1]. Common causes of inaccessible papilla include advanced locoregional obstruction or altered anatomy due to prior surgery.
Percutaneous transhepatic biliary drainage (PTBD) is an effective biliary drainage technique that provides an alternative treatment option when ERCP fails. However, PTBD is associated with rates of adverse events (AEs) between 13 to $60 \%[2,3]$. This is due to the risks of accidental dislocation of an external drainage tube and recurrent infections, which have a significant negative impact on quality of life. From a patient standpoint, the possibility of internal drainage is appealing due to convenience and likely improved quality of life, which is supported by a multicenter survey in which more than $80 \%$ of patients preferred EUS-BD over PTBD [4]. Historical surgical biliary bypass is 
seldom used as the preferred treatment option because it is associated with higher morbidity and mortality $[5,6]$.

Endoscopic ultrasound-guided biliary drainage (EUS-BD) is an advanced endoscopic technique that was first described in 2001 [7]. With the advent of the linear echoendoscope and various EUS-specific tools including lumen-apposing metal stents (LAMS), patients with failed ERCP have increasingly been offered EUS-BD to gain biliary access [8]. There are conceptual advantages associated with the technique, including the minimally invasive nature of the procedure and single-session ERCP/EUS procedure, thus reducing procedural duplicity and expediting post-procedural recovery. The advent of an electrocautery-enabled delivery system (ECE-LAMS) has further evolved the procedure into a single-step, exchange-free EUSguided puncture and deployment. The aim of this study was to evaluate technical efficacy and clinical safety of EUS-guided biliary drainage using the ECE-LAMS.

\section{Patients and methods}

We performed a retrospective analysis of prospectively collected data from all patients who underwent EUS-BD after failed ERCP from August 2016 through to June 2020. Patients underwent EUS-guided placement of a biliary stent, via a trans-duodenal approach, using the cautery-enabled LAMS system (Hot AXIOS, Boston Scientific). The procedures were classified as either choledochoduodenostomy (CDS) or cholecystoenterostomy for GBD. The study was approved by the institutional review board and granted exemption by the Health and Disability Ethics Committee.

\section{Technical Description}

All EUS-BD cases were performed under general anesthesia in a single endoscopy session, by a single endoscopist with high level of experience in therapeutic EUS procedures and in using ECE-LAMS. CDS was the preferred technique unless it could not be performed safely, defined by either the lack of a vessel-free window due to intervening vasculature or common bile duct (CBD) diameter $<10 \mathrm{~mm}$.

The bile duct was accessed with EUS localization, via a transduodenal approach. A Doppler signal was used to identify a vessel-free window and the initial puncture was made with an electrocautery tip (ERBE setting: Autocut 80-100, Effect 5). The device was also preloaded with a 0.035 guidewire to allow immediate placement of the guidewire after initial puncture. Once the catheter tip was fully inside the CBD, the distal flange was deployed under EUS guidance ( $\triangleright$ Fig. 1a). Then the catheter was retracted and "snugged" against the proximal wall of the CBD to create wall apposition. The proximal flange was deployed within the working channel of the echoendoscope, which was then slowly withdrawn with simultaneous gentle advancement of the catheter to ensure intra-duodenal deployment of the proximal flange. Finally, the correct position of the LAMS was endoscopically confirmed with visualization of bile flow through the stent ( $\mathbf{F i g} \mathbf{1} \mathbf{1 b}$ ). EUS-GBD was performed if CDS was not technically feasible. This was achieved by locali-

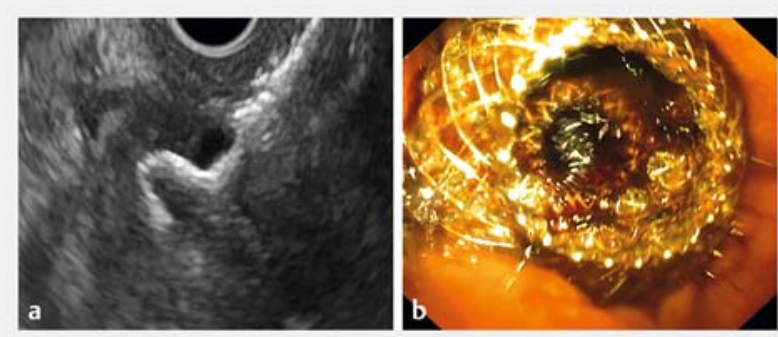

- Fig. 1 a Deployment of the distal flange under ultrasonographic guidance. b Endoscopic confirmation of successful deployment of the proximal flange and visualization of bile drainage.

zation of the gallbladder as the target puncture site, with similar techniques applied as described for CDS.

\section{Outcome measurement}

Patient demographics were reviewed, including age, sex, referral specialty, procedural indication, size of LAMS, total bilirubin (pre- and post-procedure), early and delayed adverse events (AEs), need for reintervention, and overall survival during the study period. Primary endpoints included technical and clinical success. Technical success was defined as achieving successful transluminal placement of bilioenteric fully-covered stents. Clinical success was defined as a reduction in absolute bilirubin $>50 \%$ at any time point. In addition, early confirmation of effective biliary drainage was defined by a reduction in bilirubin of $\geq 50 \%$ within 2 weeks of EUS-BD. Secondary endpoints included rate of AEs, need for reintervention, and stent patency. Stent patency was defined as absence of recurrent biliary obstruction post EUS-BD, or until patient death secondary to underlying malignant disease. Early ( $<30$ days) and late ( $>30$ days) AEs included procedure-related bleeding, reflux or stent-related cholangitis, stent occlusion, and patient death.

\section{Results}

\section{Patient demographics}

A total of 60 patients underwent EUS-BD using an AXIOS LAMS after failed ERCP with a median age of 76 years. Men represented $57 \%$ of the cohort while women represented $43 \%$. Indications for performing EUS-BD included primary and metastatic malignancies. Baseline characteristics are listed in $>$ Table 1.

\section{Technical outcome}

Fifty-six patients underwent EUS-CDS while four EUS-GBD were performed due to lack of "vessel-free" window. The mean diameter of CBD was $17 \mathrm{~mm}$ (10-33). An 8-mm LAMS was used in $71.7 \%$ of cases $(43 / 60)$ while a $10-\mathrm{mm}$ LAMS was used in $28.3 \%(17 / 60)$. Technical success was achieved in $100 \%$ of cases. One case of stent malposition was encountered during initial deployment and was salvaged immediately by stent-instent placement, resulting in successful biliary drainage via the LAMS without subsequent adverse clinical events. A total of 14 patients $(23.3 \%)$ had concomitant insertion of duodenal stents 
- Table 1 Baseline characteristics.

\begin{tabular}{|l|l|}
\hline Age & \multicolumn{1}{l}{76} \\
\hline Median & 90 \\
\hline Maximum & 52 \\
\hline Minimum & \\
\hline Sex (\%) & $34(57 \%)$ \\
\hline - Male & $26(43 \%)$ \\
\hline - Female & \\
\hline Referral (\%) & $52(86.6 \%)$ \\
\hline - Surgery & $5(8.3 \%)$ \\
\hline - Oncology & $1(1.7 \%)$ \\
\hline - Hematology & $1(1.7 \%)$ \\
\hline - Medicine & $1(1.7 \%)$ \\
\hline - Palliative care & \\
\hline
\end{tabular}

\begin{tabular}{|l|l|}
\hline Indication, $\mathbf{n}$ & 47 \\
\hline - Pancreatic cancer & 3 \\
\hline - Cholangiocarcinoma & 2 \\
\hline - Lymphoma & 2 \\
\hline - Duodenal cancer & 6 \\
\hline - Metastatic cancer & 1 \\
\hline - Colon & 2 \\
\hline - Breast & 1 \\
\hline - HCC & 1 \\
\hline - Melanoma & 1 \\
\hline
\end{tabular}

along with EUS-BD. A summary of technical characteristics is shown in $>$ Table 2.

\section{Clinical outcome}

Clinical success, determined by improvement of liver function in bilirubin, was observed in $91.7 \%$ of patients with a mean bilirubin of $202 \mu \mathrm{mol} / \mathrm{L}$ (normal $<20 \mu \mathrm{mol} / \mathrm{L}$ ) pre EUS-BD and $63.8 \mu \mathrm{mol} / \mathrm{L}$ post EUS-BD, representing a reduction of $68.4 \%$. Twenty-two patients had bilirubin value recorded on Day 3 (mean bilirubin $86 \mu \mathrm{mol} / \mathrm{L}$ ) while 11 patients had Day 7 bilirubin (mean bilirubin $43.5 \mu \mathrm{mol} / \mathrm{L}$ ). Twenty-one patients (35\%) had bilirubin value recorded at 2 weeks after EUS-BD. Normal bilirubin value was observed in 6/21 (40\%) at 2 weeks with 20 of 21 patients (95.2\%) achieving $>50 \%$ reduction in absolute bilirubin value at 2 weeks post EUS-BD and a mean reduction of $75 \%$ ( $\triangleright$ Table 3$)$.
- Table 2 Technical characteristics.

\begin{tabular}{|c|c|}
\hline Failed ERCP (\%) & 100 \\
\hline Procedure & $(n=60)$ \\
\hline - CDS & 56 \\
\hline - GBD & 4 \\
\hline Mean CBD diameter (mm) & $17(10-33)$ \\
\hline \multicolumn{2}{|l|}{ Stent size $(n)$} \\
\hline - $8 \mathrm{~mm}$ & $43(71.7 \%)$ \\
\hline - $10 \mathrm{~mm}$ & $17(28.3 \%)$ \\
\hline Reintervention & $9(15 \%)$ \\
\hline Duodenal stenting & $14(23.3 \%)$ \\
\hline \multicolumn{2}{|c|}{$\begin{array}{l}\text { ERCP, endoscopic retrograde cholangiopancreatography; } \\
\text { CDS, choledochoduodenostomy; GBD, gall bladder } \\
\text { drainage; CBD, common bile duct }\end{array}$} \\
\hline
\end{tabular}

- Table 3 Clinical outcome.

Liver function improvement $(n=60)$

\begin{tabular}{|c|c|}
\hline - Yes & $55(91.7 \%)$ \\
\hline - No & $5(8.3 \%)$ \\
\hline \multicolumn{2}{|l|}{ Total bilirubin ( $\mu \mathrm{mol} / \mathrm{L})$} \\
\hline - Pre EUS-BD & $202(30-719)$ \\
\hline \multirow[t]{2}{*}{ - Post EUS-BD } & $63.8(3-323)$ \\
\hline & $(P<.001)$ \\
\hline Day 3 bilirubin, (n) & $22 / 60(36.7 \%)$ \\
\hline - Mean bilirubin $(\mu \mathrm{mol} / \mathrm{L})$ & 86 \\
\hline Day 7 bilirubin, (n) & $11 / 60(18.3 \%)$ \\
\hline - Mean bilirubin ( $\mu \mathrm{mol} / \mathrm{L})$ & 43.5 \\
\hline Week 2 bilirubin, (n) & $21 / 60(35 \%)$ \\
\hline - Mean bilirubin ( $\mu \mathrm{mol} / \mathrm{L})$ & 46.7 \\
\hline Patient mortality & $48 / 60(80 \%)$ \\
\hline - Mean survival time after EUS-BD & 177 days \\
\hline
\end{tabular}

EUS-BD, endoscopic ultrasound-guided biliary drainage

\section{Adverse events}

The total AE rate was $20 \%$ (12/60). Early AEs (<30 days) were observed in seven patients with one case of maldeployment and six cholangitis events. Stent-related cholangitis were due to stent occlusion, and in all patients, reintervention with placement of seven French $\times 4 \mathrm{~cm}$ double pigtail stents within the LAMS to facilitate effective biliary drainage was successful. In addition, four patients developed stent occlusion as a delayed $\mathrm{AE}$ (>30 days), which also required reintervention. The overall stent occlusion rate was $16.7 \%$ with a mean procedure to stent occlusion time of 46 days (median 21 days; $3-133$ 


\begin{tabular}{|l|l|}
\hline Table 4 Adverse events. & 12 \\
\hline Total AE, $\mathrm{n}$ & 7 \\
\hline Early (<30 days) & 6 \\
\hline - Stent related cholangitis & 1 \\
\hline - Stent maldeployment & 5 \\
\hline Late (>30 days) & 4 \\
\hline - Stent occlusion - food impaction & 1 \\
\hline - Stent migration & 10 \\
\hline Stent occlusion, total & \\
\hline Time & $(6)$ \\
\hline - Early (<30 days) & $(4)$ \\
\hline - Late (>30 days) & $(8)$ \\
\hline Type & $(2)$ \\
\hline - Food impaction & $46(3-133)$ \\
\hline - Stone/sludge & 9 \\
\hline Mean time to stent occlusion, days & \\
\hline Reintervention & \\
\hline AE, adverse event. & \\
\hline
\end{tabular}

days). Nine patients underwent reintervention to rectify stent occlusion. Stent migration occurred in one patient with gall bladder drainage at 5 months after EUS-GBD. No significant stent-related bleeding had been recorded. $>$ Table 4 provides a breakdown of the types of AEs encountered as well as A stent occlusion sub-analysis.

\section{Follow up}

The mean follow-up was 7.9 months. Stent patency was recorded in $83.3 \%$ of patients $(50 / 60)$ at the end of the follow-up period. There was no observed procedure or stent-related mortality. During the study period, 48 patients died (80\%) with a mean survival time of 177 days post EUS-BD. Overall survival is shown in the Kaplan-Meier curve in $>$ Fig. 2.

\section{Discussion}

In recent years, there has been an increasing body of evidence supporting high rates of safety and efficacy for EUS-BD in the short and medium term and technical success rates of $88 \%$ to $100 \%$ and clinical efficacy rates up to $97 \%$ [9-11]. The outcome of our study is consistent with other published studies using the same ECE-LAMS (Hot AXIOS, Boston Scientific, Massachusetts, United States). EUS-BD is associated with higher clinical success and lower AE and reintervention rates compared to PTBD [12]. However, there remains much debate about its role as first-line therapy rather than ERCP-BD. Recent meta-analyses that reviewed randomized trials comparing EUS-BD and ERCP-BD have shown no appreciable difference in technical and clinical success between the two treatment modalities $[13,14]$. Fur-

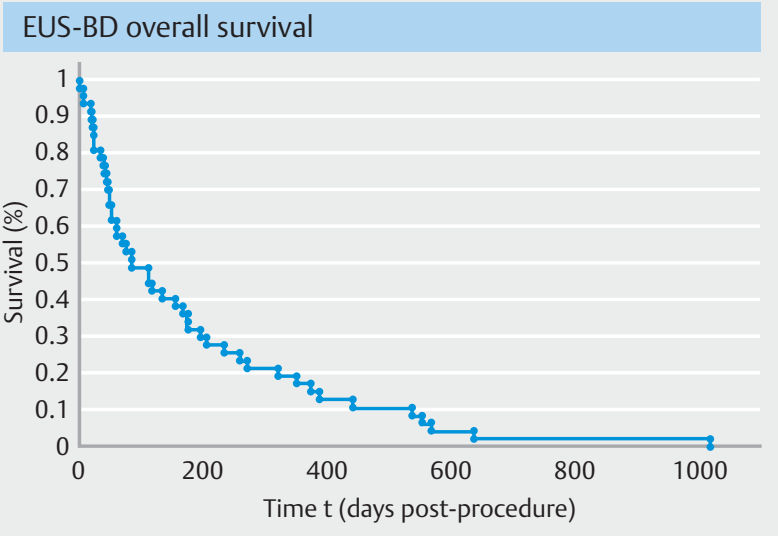

-Fig. 2 Kaplan-Meier curve of overall patient survival.

thermore, overall AE rates are lower for EUS-BD, including decreased risk of stent dysfunction (RR, 0.39), when compared with ERCP or PTBD [14]. One distinct advantage observed in EUS-BD is the significantly reduced rate of post-procedural pancreatitis, which is traditionally associated with ERCP (RR, $0.12)[14,15]$. Paik et al also reported lower rates of reintervention and higher stent patency, and therefore, improved patientreported outcome measures in the EUS-BD cohort [15].

Our observational study has demonstrated high technical success (100\%) and clinical efficacy (91.7\%) for EUS-BD using ECE-LAMS in the setting of MBO and prior failed ERCP. We observed a mean bilirubin reduction of $68.4 \%$ across the study cohort. Furthermore, those who had bilirubin monitored experienced a reduction of $>50 \%$ within the first 2 weeks, achieving a mean reduction of $75 \%$. Therefore, our data demonstrate effective biliary drainage with both CDS and GBD. Most of the cases were performed with preloading of guidewire in the LAMS delivery system to allow for successful salvage procedure in case of potential maldeployment. This occurred in one case and rescue was immediate with placement of a tubular biliary stent across the newly created transduodenal fistula. An important technical consideration is site selection for stent deployment. The mean CBD diameter was $17 \mathrm{~mm}$ in our study (10$33 \mathrm{~mm}$ ). A more dilated CBD means there is more space to allow the first flange of the stent to be deployed safely. Conventionally, the diameter of the CBD should be at least $10 \mathrm{~mm}$ to allow safe deployment of LAMS. In addition, we also found that "snugging" the deployed distal flange against the ductal wall, then deploying the proximal flange inside the delivery system prior to withdrawing the system ensures the correct positioning of the stent and minimizes maldeployment. One of the accepted indications for EUS-GBD is its application as an alternative to EUS-CDS in achieving endoscopic biliary drainage [16]. Technical success was achieved in all four patients who underwent GBD. EUS-GBD is a safe and effective endoscopic drainage option when CDS is not feasible.

The observed total AE rate in our study was $20 \%$ (12/60). This is similar to previous reports in various meta-analyses involving a heterogeneous cohort of stenting system $[17,18]$. Im- 
portantly, the AEs observed in our study were mild and did not result in prolonged hospitalization. This is favorable when compared to a surgical CDS with a morbidity rate of $30 \%$ [19]. One case of stent maldeployment was encountered in our study. This was due to maldeployment of the proximal flange with an $8-\mathrm{mm}$ stent into a $10-\mathrm{mm}$ CBD. This was immediately rectified by insertion of a $4-\mathrm{cm}$ fully-covered, self-expanding stent through the lumen of the AXIOS stent to achieve effective biliary drainage, without further complications post procedure. It is worth noting that most of the AEs observed in our study were related to stent occlusion, either early (<30 days) or delayed (>30 days). The likely reason for cholangitis and delayed stent occlusion is related to the non-coaxial drainage achieved by the EUS-CDS. Historically coaxial placement of biliary stents with ERCP presented again with cholangitis due to stent occlusion (plastic vs self-expanding metal stent [SEMS] $21 \%$ vs $8 \%$ ) [20]. Importantly, we did not encounter procedure- or stentrelated hemorrhage in our cohort. Furthermore, there was no reported bile leakage or associated peritonitis in our study. The observed reintervention rate of $15 \%$ was predominantly related to occluded LAMS. Overall, the recorded mortality rate and survival time of just under 6 months in our study are consistent with other studies involving endoscopic management of $\mathrm{MBO}$ in patients with locally advanced or metastatic malignancies [21-23]. We did not observe any fatality related to EUS-BD.

The overall observed stent patency rate was $83.3 \%$ during the study period. It is worth noting that all of the stent occlusion events were related to food impaction. Six of the 10 cases of stent occlusion were associated with the 8-mm LAMS. We did not observe stent occlusion related to stent ingrowth, overgrowth, or migration. The encountered occlusions were effectively managed with insertion of plastic pigtail stents to facilitate ongoing biliary drainage. Most stent occlusions occurred in the early period of the study ( $<30$ days). One US multicenter, retrospective analysis of EUS-CDS using ECE-LAMS demonstrated a significant reduction in rates of stent occlusion (50\% to $11.8 \%$ with preemptive placement of an axis-orienting stent through the LAMS [24]. This highlights the notion that LAMS can occlude early, and therefore, lends credit to prophylactic placement of axis-orienting stents through the LAMS lumen to prevent stent occlusion. There was also an association of LAMS obstruction in patients with concurrent duodenal obstruction [9]. In such scenario, one can also consider placement of a prophylactic axis-orientating double pigtail stent through the internal lumen of the LAMS at the time of initial deployment. We instituted routine prophylactic insertion of axis-orienting stents through the LAMS lumen in a more recent cohort of 10 patients undergoing EUS-BD. To date, we have yet to observe stent occlusion since this change in practice.

A unique clinical scenario in which EUS-BD may be preferentially selected as the primary treatment modality over ERCP-BD is in patients with concomitant gastric outlet obstruction. Though technically feasible, accessing the ampulla with a prior placed duodenal SEMS can be cumbersome, especially in type II obstruction. Achieving an appropriate endoscopic position can often be challenging, limiting the view of the ampulla. Previous studies have demonstrated significant variability in technical success of ERCP performed through existing duodenal stents, with papillary cannulation rates between 34\% and 85\% [21, 25]. Conversely, single-session EUS-BD followed by duodenal SEMS deployment may be preferable and confers a higher success rate $[9,26,27]$. One of the major advantages of using ECELAMS is that it allows a single-step puncture to gain access to the biliary system, without the need for wire system exchanges, which confers a practical benefit in reducing the length of procedures. Anderloni et al reported reduced procedural time to 30 minutes in EUS-CDS while another study by Dollhopf et al reported a mean stent deployment time of just 3.1 minutes during EUS-GBD with direct access to the gallbladder [9].

To date, EUS-BD has been performed predominantly in patients with unresectable or advanced malignancy. However, the status quo is being challenged. Fabbri et al reported a small case series suggesting EUS-BD can be used safely as a bridge to surgical interventions such as pancreaticoduodenectomy (Whipple procedure) without significantly affecting outcomes [28]. Jacques et al also reported four patients who underwent successful pancreaticoduodenectomy after EUS-CDS and placement of LAMS [29]. We propose that selection of a first-line treatment modality should be based on clinical scenarios, ductal and luminal anatomy, and local expertise. Where possible, endoscopic interventions for MBO should be performed by expert endoscopists with significant experience in both advanced ERCP maneuvers and interventional EUS.

Our study does carry inherent limitations in that this was a retrospective analysis of patients who underwent EUS-BD in a single tertiary referral center. Our study was not designed to allow direct comparison between different treatment modalities including PTBD and EUS-guided biliary drainage using a nonLAMS stent. All procedures were performed by a single endoscopist with a high level of expertise in therapeutic EUS procedures and use of LAMS. Thus, our results may lack generalizability. However, it is our opinion that such a complex procedure should be performed only by experienced endoscopists in specialized centers with therapeutic EUS capability. We were not able to assess bilirubin response post procedure in every patient in a protocolized manner due to the retrospective nature of our study. A significant portion of our study population was referred from other institutions where the local referring physicians have varying practice in terms of monitoring for treatment response. These patients were transferred back to the referring institution once they recovered from the procedure.

\section{Conclusion}

In conclusion, EUS-BD performed with ECE-LAMS is a highly effective and safe treatment for patients who present with MBO. It can be performed in a single endoscopy session after failed ERCP. AE and reintervention rates are low and can be effectively managed conservatively. We believe that our findings will add to the currently growing evidence on the safety and efficacy of EUS-BD with ECE-LAMS, and will further support the use of EUS$\mathrm{BD}$ as a safe and effective treatment option for a subset of patients with $\mathrm{MBO}$ in whom biliary cannulation cannot be achieved through conventional ERCP. However, larger random- 
ized studies are needed to compare different drainage methods to produce more robust evidence and guide practice.

\section{Competing interests}

The authors declare that they have no conflict of interest.

\section{References}

[1] Sassatelli R, Cecinato P, Lupo M et al. Endoscopic ultrasound-guided biliary drainage for malignant biliary obstruction after failed ERCP in low performance status patients. Dig Liver Dis 2020; 52: 57-63

[2] Zhao X-q, Dong J-h, Jiang K et al. PTBD vs EBD for biliary obstruction. Digestive Endoscopy 2015; 27: 137-145

[3] Nennstiel S, Weber A, Frick G et al. Drainage-related complications in percutaneous transhepatic biliary drainage: An analysis over 10 years. J Clin Gastroenterol 2015; 49: 764-770

[4] Nam K, Kim DU, Lee TH et al. Patient perception and preference of EUS-guided drainage over percutaneous drainage when endoscopic transpapillary biliary drainage fails: An international multicenter survey. Endosc Ultrasound 2018; 7: 48-55

[5] Artifon EL, Sakai P, Cunha JE et al. Surgery or endoscopy for palliation of biliary obstruction due to metastatic pancreatic cancer. Am J Gastroenterol 2006; 101: 2031-2037

[6] Bliss LA, Eskander MF, Kent TS et al. Early surgical bypass versus endoscopic stent placement in pancreatic cancer. HPB (Oxford) 2016; 18: 671-677

[7] Giovannini M, Moutardier V, Pesenti C et al. Endoscopic ultrasoundguided bilioduodenal anastomosis: A new technique for biliary drainage. Endoscopy 2001; 33: 898-900

[8] Itoi T, Binmoeller KF. EUS-guided choledochoduodenostomy by using a biflanged lumen-apposing metal stent. Gastrointest Endosc 2014; 79: 715

[9] Anderloni A, Fugazza A, Troncone E et al. Single-stage EUS-guided choledochoduodenostomy using a lumen-apposing metal stent for malignant distal biliary obstruction. Gastrointest Endosc 2019; 89: 69-76

[10] Jacques J, Privat J, Pinard F et al. Endoscopic ultrasound-guided choledochoduodenostomy with electrocautery-enhanced lumen-apposing stents: A retrospective analysis. Endoscopy 2019; 51: 540-547

[11] Tsuchiya T, Teoh AYB, Itoi T et al. Long-term outcomes of EUS-guided choledochoduodenostomy using a lumen-apposing metal stent for malignant distal biliary obstruction: A prospective multicenter study. Gastrointest Endosc 2018; 87: 1138-1146

[12] Sharaiha RZ, Khan MA, Kamal F et al. Efficacy and safety of EUS-guided biliary drainage in comparison with percutaneous biliary drainage when ERCP fails: a systematic review and meta-analysis. Gastrointest Endosc 2017; 85: 904-914

[13] Han SY, Kim S, So $\mathrm{H}$ et al. EUS-guided biliary drainage versus ERCP for first-line palliation of malignant distal biliary obstruction: A systematic review and meta-analysis. Sci Rep 2019; 9: 16551
[14] Miller CS, Barkun AN, Martel M et al. Endoscopic ultrasound-guided biliary drainage for distal malignant obstruction: a systematic review and meta-analysis of randomized trials. Endosc Int Open 2019; 7: E1563-E1573

[15] Paik WH, Lee TH, Park DH et al. EUS-guided biliary drainage versus ercp for the primary palliation of malignant biliary obstruction: a multicenter randomized clinical trial. Am J Gastroenterol 2018; 113: 987-997

[16] Imai H, Kitano M, Omoto S et al. EUS-guided gallbladder drainage for rescue treatment of malignant distal biliary obstruction after unsuccessful ERCP. Gastrointest Endosc 2016; 84: 147-151

[17] Khan MA, Akbar A, Baron TH et al. Endoscopic ultrasound-guided biliary drainage: a systematic review and meta-analysis. Dig Dis Sci 2016; 61: 684-703

[18] Wang K, Zhu J, Xing L et al. Assessment of efficacy and safety of EUSguided biliary drainage: A systematic review. Gastrointest Endosc 2016; 83: 1218-1227

[19] Kadaba R, Bowers K, Khorsandi S et al. Complications of biliary-enteric anastomoses. Ann R Coll Surg Eng 2017; 99: 210-215

[20] Sawas T, Al Halabi S, Parsi MA et al. Self-expandable metal stents versus plastic stents for malignant biliary obstruction: a meta-analysis. Gastrointest Endosc 2015; 82: 256-267.e7

[21] Staub J, Siddiqui A, Taylor L et al. ERCP performed through previously placed duodenal stents: a multicenter retrospective study of outcomes and adverse events. Gastrointest Endosc 2018; 87: 1499-1504

[22] Lee J], Hyun J], Choe JW et al. Endoscopic biliary stent insertion through specialized duodenal stent for combined malignant biliary and duodenal obstruction facilitated by stent or PTBD guidance. Scand J Gastroenterol 2017; 52: 1258-1262

[23] Mutignani M, Tringali A, Shah SG et al. Combined endoscopic stent insertion in malignant biliary and duodenal obstruction. Endoscopy 2007; 39: 440-447

[24] El Chafic AH, Shah JN, Hamerski C et al. EUS-guided choledochoduodenostomy for distal malignant biliary obstruction using electrocautery-enhanced lumen-apposing metal stents: first US, multicenter experience. Digest Dis Sci 2019; 64: 3321-3327

[25] Khashab MA, Valeshabad AK, Leung W et al. Multicenter experience with performance of ERCP in patients with an indwelling duodenal stent. Endoscopy 2014; 46: 252-255

[26] Anderloni A, Buda A, Carrara S et al. Single-session double-stent placement in concomitant malignant biliary and duodenal obstruction with a cautery-tipped lumen apposing metal stent. Endoscopy 2016; 48: E321-E322

[27] Anderloni A, Fugazza A, Auriemma F et al. Cautery-tipped lumen apposing metal stent placement through the mesh of an indwelling duodenal self-expanding metal stent. Am J Gastroenterol 2018; 113: 644

[28] Fabbri C, Fugazza A, Binda C et al. Beyond palliation: using EUS-guided choledochoduodenostomy with a lumen-apposing metal stent as a bridge to surgery. J Gastrointest Liver Dis 2019; 51: e126

[29] Jacques J, Privat J, Pinard F et al. EUS-guided choledochoduodenostomy using electrocautery enhanced lumen-apposing metal stents: a French multicenter study after implementation of the technique (with video). Gastrointest Endosc 2020; 92: 134-141 